\title{
Mécanique des fluides et applications industrielles Rapport Général
}

\author{
Fluid mechanics and industrial applications : general report \\ par R. Canavelis \\ Président de la Division "Applications Industrielles de la Mécanique des Fluides de la S.H.F."
}

This text is a synthesis of the papers presented at the XXVth Hydraulics Joumeys of the SHF in the context of the first Topic : " Fluid mechanics and industrial applications".

\section{U UNE “ ÉCOLE FRANÇAISE DE L'EAU " EN MÉCANIQUE DES FLUIDES APPLIQUÉE ?}

Le titre de ce congrès est-il applicable entre autres à la mécanique des fluides appliquée à l'hydrotechnique ?

Il est vrai que le territoire national comporte suffisamment de glaciers, de lacs, de cours d'eau et de côtes pour avoir donné naissance à de nombreuses techniques hydrauliques (telle la pompe hélice à axe vertical en bois exposée au musée du Conservatoire National des Arts et Métiers, mise au point par les gaulois et utilisée par les contemporains d'Asterix) dont est issue l'hydrotechnique d'aujourd'hui.

Il est vrai que d'illustres physiciens et mécaniciens des fluides français ont jalonné ces derniers siècles et permis d'aboutir à un enseignement de la mécanique des fluides encore très vivant dans les programmes universitaires.

Mais, comparativement à d'autres pays, la France s'est trouvée, en plus, dotée de structures fédératrices qui ont pleinement favorisé le rapprochement des hommes et des idées en hydrotechnique. On peut citer à cet égard :

- Les grands Corps d'Etat (Ponts et Chaussées, Eaux et Forêts...) et les grandes entreprises nationalisées (E.D.F., C.N.R., C.E.A. ...) qui ont pu, du fait de leur dimension, fédérer et harmoniser les compétences nationales disséminées dans les entreprises et les universités en les orientant vers des objectifs communs en vue de grandes réalisations hydrauliques. Ce fut sans nul doute le cas pour l'ensemble des ouvrages et installations hydrauliques des centrales hydrauliques, thermiques et nucléaires du parc français ou pour la mise en place et l'exploitation des grands périmètres d'irrigation.

- La Société Hydrotechnique de France qui, depuis le début du siècle, n'a cessé d'être un point de rencontre et d'échanges entre universitaires et industriels, entre constructeurs, installateurs et utilisateurs.

Les groupes de travail, au sein des divisions " Mécanique des Fluides " et " Applications Industrielles de la Mécanique des Fluides " ont réellement conduit à un enrichissement mutuel de tous leurs membres en assurant entre eux une mise en commun permanente des connaissances scientifiques et techniques du moment.

- Le Centre Technique des Industries Mécaniques (CETIM) qui est au service des industriels depuis plusieurs décennies et qui, à ce titre, apporte son soutien aux constructeurs de matériel hydraulique soit au travers de grands programmes de recherche collective soit par des actions de développement spécifiques.

\section{II $\square$ INTRODUCTION AUX COMMUNICA- TIONS DE LA QUESTION 1}

Les communications présentées par les différents auteurs sous le thème général de la mécanique des fluides et de ses applications industrielles ont été regroupées dans trois chapitres :

\section{- Etudes et Recherches}

Ce chapitre présente des méthodes d'études en indiquant le progrès des connaissances physiques et des modélisations utilisées. Les domaines abordés par les auteurs sont :

- Les instabilités dues à la cavitation dans les turbomachines.

- Prédiction de l'érosion de cavitation.

- Comportement dynamique des joints liquides annulaires ou labyrinthes dans les turbomachines. 
- La modélisation numérique d'écoulements complexes.

- La modélisation physique des ouvrages hydrauliques.

\section{- Machines Hydrauliques}

Ce chapitre présente quelques développements récents dans la conception, la réalisation et l'exploitation de grandes turbomachines telles que :

- Les pompes à volute en béton.

- Les pompes turbines DERIAZ.

- Les turbines de haute chute.

\section{- Les Grands Ensembles}

Ce chapitre traite de la maîtrise des problèmes d'ensemble dans les installations complexes en s'attachant tout particulièrement à l'optimisation des systèmes et de leur exploitation dans les domaines suivants :

- Fiabilité des stations de pompage et des réseaux de distribution.

- Maitrise du comportement d'ensemble des centrales hydroélectriques.

- Régulation des aménagements hydrauliques fluviaux.

\section{III $\square$ ETUDES ET RECHERCHES}

\section{- 3.1. Les instabilités dues à la cavitation dans les turbo- machines.}

La cavitation constitue une limite physique d'utilisation des turbomachines hydrauliques. Elle devient un phénomène critique dans les systèmes industriels qui comportent des turbomachines " poussées " c'est-à-dire à hautes performances, avec trois risques essentiels :

- La chute de performances.

- L'érosion des parois solides en contact avec l'écoulement cavitant.

- Les instabilités hydromécaniques : fluctuations de régime, vibrations, bruit.

Ce dernier aspect est souvent le plus difficile à appréhender car il fait intervenir des figures de cavitation très développées dont la compréhension nécessite une schématisation d'écoulements fortement biphasiques avec prise en compte d'interactions fluide-structure et machine-circuit.

Parmi les applications industrielles pointues mises en œuvre en France ces dernières décennies et soumises à ce type d'écoulement on peut citer :

- Les pompes alimentaires des centrales nucléaires de $1500 \mathrm{MW}$.

- Les pompes d'injection de sécurité moyenne pression (I S M P) de ces mêmes centrales dont le premier étage est constitué d'une pompe centrifuge multiétagée avec hélice de gavage (ou inducteur).

- Les pompes cryogéniques du lanceur de type ARIANE qui sont des pompes axiales multicellulaires avec inducteur.

Compte tenu de leur grande vitesse de rotation liée à la recherche d'une compacité maximale, ces pompes ont besoin d'une capacité d'aspiration poussée (d'un faible NPSH requis). Mais elles doivent également pouvoir fonctionner en toute fiabilité dans une plage de débit aussi large que possible, d'où un risque important d'instabilités de fonctionnement, notamment dans les zones hors adaptation.

La communication $\mathrm{N}^{\circ} 1$ relative à la " prédiction de l'apparition de la cavitation dans un inducteur placé à l'amont d'une roue centrifuge " présente les études expérimentales menées au Laboratoire de Mécanique des Fluides de l'INSA de Lyon.
L'observation fine de l'évolution des figures de cavitation et des grandeurs caractéristiques de l'écoulement s'avère indispensable pour mieux comprendre le mécanisme des instabilités. Elle permet en outre d'établir une cartographie précise des zones de fonctionnement admissibles dans le plan débit-NPSH.

L'optimisation du tracé de ces machines ne pourra par ailleurs être assurée de manière industrielle que si l'on dispose un jour d'un outil numérique fiable de schématisation. Les calculs réalisés par le Laboratoire de l'Ecole Centrale de Lyon montrent les progrès réalisés dans cette voie.

Les développements en cours permettent d'espérer que l'on pourra dans un avenir proche augmenter la vitesse de rotation et la compacité d'un plus grand nombre de pompes industrielles grâce à une meilleure maitrise de la cavitation développée, dans une large plage de fonctionnement et, ceci, grâce notamment à une extension de l'utilisation de l'inducteur.

\section{- 3.2 La prédiction de l'érosion de cavitation.}

Dans les turbomachines poussées, les risques d'érosion par cavitation peuvent limiter encore plus le dimensionnement et les conditions extrêmes de fonctionnement que les risques de chute de performance ou d'instabilité. En effet, l'érosion peut se manifester dès l'apparition des premières poches ou bulles de cavitation si les conditions locales de vitesse ou de pression s'avèrent défavorables.

On peut dire que l'érosion de cavitation est en particulier un frein majeur au développement de turbomachines compactes à grande vitesse de rotation ou à grande hauteur d'élévation ou de chute telles que :

- Les turbines hydrauliques de haute chute.

- Les pompes d'accumulation et pompes-turbines réversibles de grande hauteur d'élévation.

- Les pompes alimentaires de chaudières des centrales thermiques et nucléaires.

Il s'agit bien d'un enjeu économique important puisque l'érosion des matériaux peut conduire à des arrêts de production ainsi qu'à des coûts de maintenance élevés. Cela explique les efforts déployés en France depuis plusieurs décennies, notamment sous l'impulsion de grands exploitants comme Electricité de France, pour tenter de prédire les érosions au stade du projet.

La communication $\mathrm{N}^{\circ} 2$ intitulée " Erosion de cavitation : méthodes pratiques de prédiction des dégâts au stade du projet " fait la synthèse des méthodes mises progressivement au point en France au cours des dernières décennies dans différents centres de recherches qui ont lancé des programmes plus ou moins complémentaires tels que :

- GEC ALSTHOM ACB-CERG à Grenoble.

- L'Institut de Mécanique de Grenoble (I.M.G.) de l'Université de Grenoble.

- Le Centre de Recherches de CHATOU de la Direction des Etudes et Recherches d'E.D.F.

\section{- Le CETIM, centre de Nantes.}

Il est à noter que la S.H.F. a depuis longtemps favorisé le rapprochement et le dialogue de tous ces organismes industriels et universitaires au sein du Groupe de Travail " Cavitation" de la Division "Mécanique des Fluides" et a joué un rôle essentiel dans le transfert, l'analyse et la synthèse des résultats. Ainsi est-on arrivé à la méthode globale prévisionnelle présentée par les auteurs à partir de la maitrise des différentes phases intermédiaires :

- Représentation sur modèle des phénomènes de cavitation. 
- Caractérisation des érosions par la mesure des impacts.

- Application de lois de similitude à l'histogramme des impacts de cavitation permettant de modifier les dimensions, la vitesse, le liquide, le matériau.

- Génération contrôlée d'érosion à l'aide d'un simulateur.

Même si des progrès restent nécessaires dans la précision des lois de transposition et dans la facilité de mise en œuvre des méthodes d'évaluation, on dispose aujourd'hui d'une méthodologie généralisable à toutes les applications industrielles.

\section{- 3.3 Comportement dynamique des joints liquides annulaires ou labyrinthes dans les turbomachines}

Le développement, au cours des dernières décennies, des puissances unitaires des turbomachines hydrauliques et notamment des hauteurs totales d'élévation (ou de chute) et des vitesses de rotation, a rendu beaucoup plus exigeant le calcul dynamique des éléments de la ligne d'arbre.

On a dû prendre en compte de façon beaucoup plus fine les effets de palier des différents espaces liquides localisés le long de la ligne d'arbre et notamment les aspects stabilisants ou déstabilisants (effet LOMACHIN) liés à la masse ajoutée à la raideur et à l'amortissement. Les joints liquides ont donné lieu à de nombreuses modélisations décrites dans la littérature mondiale. Toutefois, dans le cas des pompes à grande hauteur d'élévation et à grande vitesse de rotation ou des turbines hydrauliques à grande hauteur de chute et de grandes dimensions, les écoulements dans ces espaces deviennent fortement turbulents. La résolution numérique des équations représentant ces écoulements devient alors complexe et les modélisations proposées exigent un calage à partir de résultats expérimentaux complets et précis.

La communication $\mathrm{N}^{\circ} 3$ relative à la " mise en place d'un dispositif expérimental de caractérisation du comportement statique et dynamique des lames fluides à grand nombre de Reynolds " décrit un projet de banc d'essai piloté par le Laboratoire de Mécanique des Solides de l'Université de Poitiers en collaboration avec plusieurs industriels français.

Ce banc d'essai sera un outil original de tout premier plan au service des industriels pour leur permettre de développer des formes nouvelles de joints liquides annulaires ou labyrinthes, d'en comprendre le fonctionnement interne et de maîtriser leur comportement dynamique dans des conditions de fonctionnement poussées.

\subsection{La modélisation numérique d'écoulements complexes}

Le dernier quart de ce siècle aura vu un accroissement constant de la capacité et de la compacité des ordinateurs avec une diminution parallèle de leur coût. Ce phénomène a entraîné une explosion de l'informatique dans tous les bureaux d'études et une multiplication des codes de calcul disponibles dans le commerce et utilisables dans des domaines d'application variés et larges. La mécanique des fluides n'a évidemment pas échappé à cette tendance et la communauté scientifique française est devenue un grand espace d'utilisation et de production de logiciels de calcul d'écoulements.

La communication $\mathrm{N}^{\circ} 4$ présente un exemple typique d'utilisation du logiciel FLUENT qui est un code de calcul largement répandu et applicable à des écoulements tridimensionnels visqueux et turbulents.

Cet exemple qui concerne la " modélisation numérique au service de la conception optimisée de réacteurs de post-chlo- ration " montre comment un tel outil permet d'optimiser l'architecture géométrique des bassins de chloration en maîtrisant la dispersion des temps de séjour de l'eau traitée, ce qui permet d'évaluer des critères de qualité d'eau exigés par la législation internationale. Cette application spécifique montre bien la flexibilité de l'outil numérique par rapport aux modélisations physiques traditionnelles.

La communication $\mathrm{N}^{\circ} 5$ intitulée " TELEMAC : système hydroinformatique " décrit les principales caractéristiques de ce logiciel mis au point au Laboratoire National d'Hydraulique de CHATOU au sein de la Direction des Etudes et Recherches d'E.D.F.

Ce logiciel dédié à la schématisation d'écoulements à surface libre est le type même d'outil numérique convivial ouvert à de multiples utilisateurs ainsi qu'à une grande diversité d'applications. Ce logiciel comprend de nombreux modules spécifiques qui permettent d'orienter une étude par les applications les plus variées allant des effets de surface dus au vent ou à la pression atmosphérique aux effets de fond tels que les transports solides et les érosions en passant par tous les effets de masse tels que marées, courants, houle, variations de densité, pollution, etc.

Le succès d'un tel logiciel peut se mesurer entre autres à sa grande diffusion dans le monde (plus de cinquante équipes scientifiques l'utilisent) et à la très large collaboration internationale qui en résulte.

\subsection{La modélisation physique des ouvrages hydrauliques}

Bien avant l'explosion des outils de modélisation numérique, l'application des lois de similitude de la mécanique des fluides a permis de représenter sur maquette des écoulements réels complexes. De nombreux laboratoires français universitaires ou industriels ont accumulé un long savoirfaire qui tient à :

- la connaissance des effets d 'échelle et des compromis à réaliser entre des critères de similitude souvent incompatibles (Reynolds, Froude, Weber ...),

- la maîtrise d'une technologie de réalisation des maquettes et d'une métrologie appropriée,

- l'expérience d'une large palette de solutions pratiques à mettre en œuvre pour optimiser le fonctionnement des ouvrages.

Beaucoup de ces laboratoires ont acquis une notoriété internationale et continuent de travailler sur des grands projets à l'échelle mondiale.

La question se pose néanmoins de savoir dans quelle situation une modélisation physique reste économiquement intéressante par rapport à une modélisation numérique.

Il semble que ce soit le cas lorsque l'étude s'applique à une géométrie complexe pour laquelle la modélisation numérique conduirait à un maillage très dense et hétérogène ; c'est souvent le cas des grands aménagements dans lesquels on doit réaliser à la fois des modifications de forme générale et des modifications de détail. Par ailleurs, les modifications de forme de détail sont en général facilement réalisables sur maquette grâce à la standardisation des composants.

Il convient toutefois de signaler qu'aujourd'hui la plupart des laboratoires d'hydraulique disposent simultanément des outils numériques et physiques et sont souvent amenés à utiliser conjointement les deux types d'outils dans une même étude, soit pour obtenir des résultats complémentaires soit pour caler le modèle numérique. 
La communication $\mathrm{N}^{\circ} 6$ relative à " l'optimisation des prises d'eau et des stations de pompage " concerne des ouvrages et installations de géométrie complexe avec de multiples composants : pertuis d'entrée, dégrillage, filtration, bassin de répartition, rues d'eau avec aspiration des pompes ; autant d'éléments qu'il serait difficile d'inclure dans un maillage suffisamment fin.

Parmi les phénomènes étudiés, les tourbillons et vortex de surface peuvent autant provenir de la géométrie d'ensemble que de détails de forme et seule l'expérience acquise par le laboratoire lui permet d'estimer l'importance des paramètres de forme significatifs. C'est aussi l'expérience qui permet d'estimer la distorsion de ces écoulements due aux effets d'échelle et au non respect absolu de certains critères de similitude.

De manière générale, il convient d'insister sur l'intérêt majeur qu'il y a à faire systématiquement l'étude sur maquette des stations de pompage au niveau du projet afin d'éviter de découvrir un dysfonctionnement irréparable au moment de la mise en route industrielle des équipements sur site. Cette communication montre en outre l'intérêt qu'il peut y avoir, dans l'étude des grands ensembles de pompage, à combiner la modélisation physique (côté prise d'eau) et la modélisation numérique (côté circuit de refoulement).

La communication $\mathrm{N}^{\circ} 7$ relative à " l'étude hydrodynamique de l'écoulement en amont d'une retenue hydraulique" présente les résultats de mesure sur maquette des vitesses moyennes et turbulentes dans la couche limite au voisinage de la vidange de fond d'une retenue.

Cette étude expérimentale permet de déterminer les différentes lois de frottement qui régissent la couche limite graduellement variée que génère l'écoulement en amont de l'orifice de vidange au cours d'une chasse. Il est clair qu'une telle étude peut constituer une étape de validation dans la mise au point d'un code de calcul.

Il convient par ailleurs de resituer cette étude dans le contexte des recherches entreprises pour mieux comprendre le processus d'évacuation des dépôts sédimentaires par système de chasses successives dans le fond des retenues hydrauliques.

La communication $\mathrm{N}^{\circ} 8$ concerne les " évacuateurs de Turkwell et de Katse " en Afrique. Elle montre comment on peut optimiser sur maquette la géométrie des ouvrages évacuateurs de grands barrages pour dissiper l'énergie disponible tout en évitant les affouillements en aval du barrage principal.

Dans un tel exemple on retrouve les avantages que peut présenter un modèle physique par rapport aux modèles numériques :

- prise en compte d'une géométrie complexe avec des dimensions très variables entre la géométrie générale et les détails de forme,

- modification aisée des formes locales,

- schématisation réaliste des entraînements d'air et des stabilités de jets et de nappes.

Ici encore, comme dans le cas des stations de pompage, l'expérience acquise par le laboratoire est fondamentale pour aboutir rapidement à une solution optimale.

De plus, la sécurité finale des ouvrages ne saurait être totalement assurée sans cette validation préalable sur maquette au niveau du projet.

\section{MACHINES HYDRAULIQUES}

\subsection{Les pompes à volute en béton}

La communication $\mathrm{N}^{\circ} 9$ intitulée " les pompes à volute en béton "BERGERON" : applications aux grands transferts d'eau" présente les récents développements d'une technique qui est née en France en 1917 et qui n'a cessé de se moderniser et d'étendre ses domaines d'application durant les récentes décennies.

Cette technique fut mise au point pendant la première guerre mondiale en raison de la rareté et du coût de l'acier pendant cette période d'hostilités.

On peut dire que cet aspect économique reste aujourd'hui un critère significatif de compétitivité à l'exportation vers des pays en voie de développement. En effet, la réalisation in situ du corps de pompe en béton présente les avantages suivants :

- limitation du nombre de composants mécaniques importés,

- utilisation d'entreprises et de main d'œuvre locale pour la réalisation du génie civil et des coffrages,

- réalisation d'une structure rigide et stable favorable à la tenue au séisme dans certains pays où des risques significatifs existent.

Il convient par ailleurs d'insister sur le très large éventail d'applications dans lesquelles cette technique peut être utilisée avec tous les avantages spécifiques à sa technologie, à chaque fois que des débits d'eau importants sont requis notamment en eau de mer (de 10 à $30 \mathrm{~m}^{3} / \mathrm{s}$ par pompe) :

- installations portuaires : forme de radoub,

- alimentation de bassins,

- réfrigération des centrales thermiques et nucléaires,

- réfrigération des grands complexes industriels,

- grands transferts d'eau brute pour l'irrigation, le drainage.

Cette grande diversité autorise à penser que ce type de pompe a encore un très bel avenir dans de nombreux pays qui doivent s'équiper avec un matériel présentant une grande fiabilité.

\subsection{Mise en œuvre de pompes-turbines DERIAZ}

La communication $\mathrm{N}^{\circ} 10$ intitulée “ NAUSSAC 2 : mise en œuvre de pompes-turbines DERIAZ " présente l'aménagement de Naussac sur le haut bassin de l'Allier. La réalisation antérieure d'un barrage a permis de constituer un réservoir amont en vue du soutien d'étiage de l'Allier et de la Loire. La nouvelle réalisation d'un bassin et d'une station de pompage en pied de barrage permettra à l'avenir, premièrement de compléter le remplissage du réservoir supérieur et secondairement dans l'autre sens, de turbiner les débits lâchés vers l'aval et de réduire ainsi pour la collectivité les coûts de fonctionnement de l'aménagement global.

Cet aménagement s'avère d'une grande originalité non seulement par son impact sur l'environnement mais également par le choix des équipements mécaniques et notamment des pompes-turbines : les trois machines de type DERIAZ de $3 \mathrm{MW}$ chacune sont en effet très particulières à la fois par leur conception hydraulique et leur technologie interne.

Le choix de cette conception hydraulique qui offre un profil méridien hélico-centrifuge à pales orientables, présente deux avantages principaux :

- possibilité de fonctionner sous des hauteurs de chute nettement supérieures à celles de turbines de type KAPLAN : ici entre 30 et $60 \mathrm{~m}$, 
- une plage étendue de fonctionnement grâce à l'adaptation des pales : ici, le débit unitaire est variable de 1 à $5,5 \mathrm{~m}^{3} / \mathrm{s}$. Cette souplesse de fonctionnement permet notamment de pomper " au fil de l'eau" en suivant les variations de débit de l'Allier.

Par ailleurs, l'objectif de non pollution de l'eau pompée ou turbinée a conduit à mettre au point des dispositifs techniques tout à fait originaux en éliminant les commandes classiques avec circuit d'huile :

- commande des pales orientables de la roue par un servomoteur à eau,

- commande des vannes du distributeur par système électrique,

- paliers de la ligne d'arbre fonctionnant avec des coussinets en céramique lubrifiés par l'eau du site.

Ces dispositifs mis au point par GEC Alsthom Neyrpic constituent une première et devraient pouvoir servir de référence à bien d'autres sites dans le monde où le respect de l'environnement devient une exigence principale.

\subsection{Les grandes turbines hydrauliques}

Le développement énergétique des grands pays à forte croissance possédant des ressources hydrauliques importantes conduit à la réalisation de machines de très grandes dimensions avec des puissances unitaires élevées. C'était déjà le cas pour le Brésil et le Paraguay en ce qui concerne l'aménagement d'Itaipu qui comportait 18 turbines d'une puissance unitaire supérieure à $700 \mathrm{MW}$. L'aménagement en cours du site des Trois Gorges sur la rivière Yangtze en Chine repousse encore ces limites avec une puissance unitaire maximale de $852 \mathrm{MW}$, un débit maximal de $1065 \mathrm{~m}^{3} / \mathrm{s}$ et une chute nette maximale de $113 \mathrm{~m}$.

La communication $\mathrm{N}^{\circ} 11$ décrit les caractéristiques principales de ce projet et des machines conçues, fabriquées et installées par GEC Alsthom Neyrpic dans le cadre de l'appel d'offre international lancé par le client.

Pour des raisons de fiabilité, le client a exigé une conception classique ayant déjà fait ses preuves. L'originalité d'un tel projet tient donc essentiellement à l'effet de taille avec toutes les conséquences sur les processus de fabrication et de montage ainsi qu'au service très particulier que constitue le transfert de technologie.

Les dimensions et les masses, jamais atteintes par le passé, ont bien évidemment nécessité de nombreux calculs de mécanique afin de valider une telle extrapolation. A titre d'exemple les diamètres d'arbre et de roue sont respectivement de $4 \mathrm{~m}$ et $10,6 \mathrm{~m}$ dans ce projet contre environ $2,5 \mathrm{~m}$ et $8 \mathrm{~m}$ à Itaipu. On devine aisément quels peuvent être les problèmes d'assemblage, d'usinage et de transport qui en résultent. Il convient également de tenir compte de l'organisation très particulière mise en place pour mâttriser une sous-traitance locale imposée la plupart du temps par le client.

Il est enfin nécessaire d'insister sur l'importance que prend dans de tels projets le transfert de technologie et sur les moyens humains et matériels mis en place pour satisfaire à une telle exigence qui se traduit par une formation documentaire et pratique de multiples équipes de techniciens pendant plusieurs années avec l'objectif final d'une construction entièrement transférée de 12 machines et l'obligation d'une mise en route satisfaisante. C'est à ce prix, qu'aujourd'hui, les constructeurs français peuvent se positionner sur un tel marché.

\section{V — GRANDS ENSEMBLES}

\subsection{Fiabilité des stations de pompage et des réseaux de distribution}

Les systèmes de pompage et de distribution se rencontrent dans les secteurs les plus divers : traitement d'eau brute et adduction d'eau potable, irrigation, drainage, eaux usées, bassins fluviaux et portuaires, formes de radoub, centrales hydrauliques, thermiques ou nucléaires, grands complexes industriels. Cette large palette d'applications reflète la grande diversité des problèmes à résoudre à l'intérieur du métier de concepteur de stations de pompage.

Toutefois, dans tous les cas, le concepteur du système doit répondre aux exigences suivantes :

- adapter au mieux le système aux conditions réelles du site : climat, topologie, nature des eaux, structure du circuit aval, conditions contractuelles de fonctionnement en tenant compte de toutes les évolutions futures envisageables (encrassement, extensions...),

- assurer la fiabilité maximale de l'ensemble car tout incident se traduit non seulement par des coûts d'intervention mais aussi par une indisponibilité des installations et, souvent, par des pertes de production importantes,

- minimiser le coût global du système non seulement au niveau de l'investissement initial mais, si possible, en tenant compte de son rendement énergétique sur une période correspondant au cycle de vie, ce qui amène à prendre en compte les coûts de consommation et de maintenance.

La communication $\mathrm{N}^{\circ} 12$ relative aux " grandes stations de pompage " dans les usines de traitement d'eau brute et de distribution d'eau potable montre bien le rôle primordial joué par l'ensemblier installateur qui doit avoir le souci permanent de vérifier la compatibilité des équipements avec les conditions réelles du site et les exigences du cahier des charges en vue d'assurer le bon fonctionnement de l'ensemble. Il apparait clairement que le maître d'EEuvre doit agir en architecte industriel et non pas simplement en entrepreneur général.

Les exemples de dysfonctionnement indiqués dans cette communication font ressortir deux types d'erreurs à éviter systématiquement au stade de la conception :

- la méconnaissance des données réelles du site : le concepteur doit valider toutes les données qui lui sont transmises et vérifier par lui-même l'état du site,

- la méconnaissance des caractéristiques réelles des équipements sous-traités aux constructeurs de matériel : le concepteur doit instaurer avec les équipementiers un dialogue ouvert, dans un climat de coopération, en communiquant bien toutes les données et toutes les attentes.

La communication $\mathrm{N}^{\circ} 13$ intitulée "Les modèles en régime permanent et en régime transitoire : des outils indispensables pour concevoir, optimiser la gestion et protéger les installations de production et de distribution d'eau " montre ce que peuvent être les contraintes de gestion d'un réseau complexe de production et de distribution d'eau potable tel que celui qui est géré aujourd'hui par le Syndicat des Eaux d'Tle-de-France. Ce réseau est d'ailleurs sans doute analogue à celui de nombre de grandes agglomérations dans le monde.

Comme dans beaucoup d'installations requérant une très grande fiabilité, l'exploitant est amené à mettre en place un système de gestion globale qui prend en compte l'ensemble des éléments statiques (conduites, réservoirs) et dynamiques (pompes, vannes, asservissements) du système afin de maîtriser toutes les situations possibles imaginables. L'outil 
informatique mis en place permet effectivement d'optimiser l'exploitation du système dans le cadre des grandes exigences mentionnées ci-dessus :

- fiabilité de la distribution en situation incidentelle,

- maintient de la qualité des eaux,

- minimisation du coût énergétique,

- adaptation à l'évolution des paramètres de consommation.

\subsection{Maîtrise du comportement d'ensemble des cen- trales hydroélectriques.}

ans tous les grands systèmes hydrauliques, au-delà de l'optimisation des caractéristiques individuelles de chaque équipement, il convient de s'assurer de la compatibilité de tous les composants entre eux et, ceci, dans tous les régimes de fonctionnement compte tenu des interactions hydrauliques, mécaniques et hydromécaniques entre machines, circuits et ouvrages.

Dans le cas particulier des installations hydroélectriques, les risques d'instabilités d'ensemble doivent être systématiquement éliminés compte tenu des puissances mises en jeu, de la dimension des pièces mécaniques sollicitées et du coût des arrêts de production.

La communication $\mathrm{N}^{\circ} 14$ relative à la " commande avancée pour la régulation des aménagements hydrauliques " montre les progrès récents réalisés dans la conception de systèmes de régulation qui font appel à la " commande robuste " à partir de modèles simplifiées du fonctionnement du système.

Les avancées méthodologiques récentes de l'Automatique semblent permettre de résoudre des études complexes de conduite et de régulation des aménagements hydrauliques soit dans le cas de la rénovation d'anciennes centrales en France soit pour de nouveaux grands projets à l'étranger.

La communication $\mathrm{N}^{\circ} 15$ intitulée " Stabilisation d'une pompe-turbine monoétagée présentant une instabilité en marche à vide " présente un problème d'instabilité hydraulique découvert sur site au cours de la mise en service d'une pompeturbine et résolu grâce à une modification conjointe du régulateur de vitesse et de la perte de charge de la conduite forcée.

Cet exemple montre que les incertitudes résiduelles de caractéristiques dues aux effets d'échelle entre la machine industrielle et le modèle réduit peuvent générer des dysfonctionnements inattendus. Il est toutefois satisfaisant de constater que les outils de simulation des transitoires hydrauliques restent un outil précieux pour la résolution de tels problèmes.

La communication $\mathrm{N}^{\circ} 16$ relative à " l'analyse et la mâ̂trise des vibrations d'une conduite forcée extérieure " décrit un cas d'interaction fluide-structure dans une conduite forcée soumise à des fluctuations de pression provenant du fonctionnement en marche à vide d'une pompe-turbine.

Cet exemple relatif à un incident découvert sur site au cours de la mise en marche industrielle s'avère intéressant par la démarche suivie. Il a en effet fallu tout d'abord caler les paramètres mécaniques de la structure à l'aide d'une analyse modale de la conduite vide, effectuée sur site. Sur la base de ces éléments on a pu ensuite effectuer un calcul des fréquences propres et des contraintes dans la conduite en charge pour définir les raidissements nécessaires et résoudre ainsi le problème.

\subsection{Régulation des aménagements hydrauliques fluviaux}

L'optimisation de la gestion des volumes d'eau le long des fleuves, notamment en période de crue, est un souci permanent des exploitants compte tenu des multiples implications : production électrique, navigation, irrigation, loisirs. Il est donc apparu du plus grand intérêt d'améliorer les procédures de gestion manuelle à effet localisé en mettant en place une régulation par commande prédictive qui tient compte du comportement détaillé des écoulements fluviaux en amont et en aval de chaque ouvrage ainsi que des tendances des évolutions futures estimées.

La communication $\mathrm{N}^{\circ} 17$ intitulée " Régulation fluviale par commande prédictive avec modèle de connaissance embarqué " présente l'exemple particulier des aménagements de la Compagnie Nationale du Rhône (C.N.R.) le long du Rhône et décrit les outils numériques mis en place en vue de la mise en œuvre d'un système de régulation par commande prédictive.

Il convient tout d'abord de noter que le logiciel de simulation des écoulements à surface libre dans un réseau quelconque de rivières et canaux développé par la C.N.R. entre tout à fait dans le cadre des outils numériques modernes dont il a été question au chapitre 3-4 de ce Rapport. Ce logiciel est ensuite utilisé comme modèle pour déterminer la tendance de l'évolution des grandeurs physiques et en déduire la meilleure commande pour suivre une trajectoire aboutissant à la consigne choisie. Les premières applications de cette méthodologie ont pu démontrer un accroissement notable d'efficacité dans les temps d'obtention des niveaux de consigne par rapport aux procédures de régulation classiques.

Enfin, une perspective essentielle apparaît, celle de pouvoir appliquer cette nouvelle méthode à plusieurs aménagements en série en vue de mettre en place une conduite centralisée pour une chaîne d'aménagement.

\section{VI — CONCLUSION GÉNÉRALE SUR LA QUESTION 1}

Les communications proposées par les différents auteurs dans le cadre de cette Question couvrent un certain nombre de domaines dans lesquels les techniciens français se trouvent en pointe ou, pour le moins, ont fait des apports originaux et significatifs. Il est néanmoins évident que la place disponible dans ces journées n'a pas permis de couvrir l'ensemble des domaines concernés.

Dans le domaine des études et recherches les chercheurs français ont sans nul doute une position de leader dans la maîtrise des phénomènes de cavitation et de leurs effets industriels. On doit reconnaître cependant que dans les domaines de la dynamique des joints liquides ou de la modélisation numérique ou physique des écoulements, il s'agit d'avantage de contributions originales complémentaires des avancées mondiales. Par ailleurs, de nombreux axes de recherche qui ont eu une place marquante dans les travaux français des deux dernières décennies n'ont pas été abordés dans ces journées. On peut citer notamment :

- l'étude de la turbulence, des couches limites et des sillages qui a été particulièrement marquante dans le domaine des réalisations navales,

- les études de thermohydraulique appliquées notamment aux écoulements dans les réacteurs nucléaires et divers circuits associés, 
- les études d'hydroélasticité et d'hydroacoustique également très poussées dans le cadre des développements de l'industrie nucléaire, des engins spatiaux ou de la propulsion navale.

Dans le domaine des machines hydrauliques, les constructeurs français ont également acquis une position privilégiée dans le cadre du développement des grandes pompes à volute en béton armé (notamment à l'occasion de la mise en œuvre des paliers nucléaires de $1000 \mathrm{MW}$ et $1500 \mathrm{MW}$ ) et des turbines hydrauliques de grande puissance. Ces journées n'ont par ailleurs pas permis d'évoquer quelques autres développements remarquables tels que :

- les pompes cryogéniques des lanceurs de type Ariane,

- le matériel de propulsion naval,
- la maîtrise des fonctionnements hors adaptation.

Dans le domaine des grands ensembles, les techniques d'optimisation présentées dans ces journées sont pour la plupart originales et fondées sur les outils d'analyse les plus récents. On aurait pu également citer des applications particulièrement intéressantes dans des secteurs tels que la propulsion navale, l'industrie nucléaire, chimique ou pétrolière.

Cette richesse de réalisations tant sur le territoire national qu'à l'exportation, et d'expériences acquises, est sans nul doute la meilleure chance que peut avoir l'hydrotechnique industrielle française pour intensifier sa participation au développement d'un monde équipé d'installations plus performantes, plus fiables et plus économiques. 